\title{
Pushing the Limits of Microfocus X-Ray Sealed Tube Sources for Biological and Chemical Crystallography
}

\author{
J Graf ${ }^{1}$, T Stuerzer ${ }^{2}$, M Benning ${ }^{3}$, H Ott ${ }^{2}$, P Radcliffe ${ }^{1}$, J Schmidt-May ${ }^{1}$, C Michaelsen ${ }^{1}$ \\ ${ }^{1}$ Incoatec GmbH, Geesthacht, Germany, ${ }^{2}$ Bruker AXS GmbH, Karlsruhe, \\ Germany, ${ }^{3}$ Bruker LLC, Madison WI, USA \\ marketing@incoatec.de
}

\begin{abstract}
The structure determination on ever smaller and weakly diffracting crystals is one of the biggest challenges in the development of in-house X-ray analytical equipment for chemical and biological crystallography, which continuously raises the requirements for modern X-ray sources and detectors. Nowadays, modern low power microfocus X-ray sealed tube sources, such as the Incoatec Microfocus Soure I $\mu \mathrm{S}$, define the state-of-the-art for most in-house X-ray diffraction equipment, as they deliver intensities in the range of rotating anodes, yet maintain all the comfort of a sealed tube system.

Throughout the past years, we have continuously improved the performance of the I $\mu \mathrm{S}$ by optimizing critical parameters in the X-ray tube and adapting the X-ray optics, making the $\mathrm{I} \mu \mathrm{S}$ the market-leading microfocus sealed tube X-ray source with more than 1000 sources sold to date world-wide. The latest improvement for the I $\mu$ S 3.0, the first and only microfocus sealed tube source fully optimized for X-ray diffraction applications, is a dedicated multilayer mirror which delivers an intensity in the range of $8 \cdot 10$ [sup]...[/sup]10 phts/s/mm[sup]...[/sup]2 with a divergence that matches the typical mosaicity of weakly diffracting chemical samples. Applications that demand an even higher brightness, such as protein crystallography, benefit from our recently introduced unique new class of microfocus sealed tube sources which uses diamond as a heat sink to cool the anode. This I $\mu$ S DIAMOND combines the performance of a modern $1 \mathrm{~kW}$ microfocus rotating anode with all the comfort of a conventional microfocus sealed tube source, and is now available for $\mathrm{Cu}-\mathrm{K} \alpha$, Mo-K $\alpha$ and $\mathrm{Ag}-\mathrm{K} \alpha$ radiation.
\end{abstract}

We will be presenting selected results showing the impact of these recent developments on the data quality.

Acta Cryst. (2020). A76, a103 